\title{
Technology Preference in Choices of Delivery Care Utilization from User Perspective -A Community Study in Vietnam
}

\author{
Tran Khanh Toan ${ }^{1, *}$, Bo Eriksson ${ }^{2}$, Pham Nhat An $^{3}$, Nguyen Thi Kim Chuc ${ }^{1}$, Goran Bondjers ${ }^{4}$, Karin Gottvall ${ }^{5}$ \\ ${ }^{1}$ Family Medicine Department, Hanoi Medical University (HMU), Hanoi, Vietnam \\ ${ }^{2}$ The Nordic School of Public Health (NHV), Gothenburg, Sweden \\ ${ }^{3}$ The National Hospital for Pediatrics, Hanoi, Vietnam \\ ${ }^{4}$ Sahlgrenska Academy, University of Gothenburg (GU), Gothenburg, Sweden \\ ${ }^{5}$ Department of Public Health Sciences, Karolinska Institutet, Stockholm, Sweden \\ *Corresponding author: tktoan@yahoo.com
}

Received January 01, 2013; Revised January 20, 2013; Accepted February 15, 2013

\begin{abstract}
Choices for delivery care are made based on the available resources and influence health outcomes of women and their children. The aim of the paper is to study utilization and preference for delivery care and related factors in one urban and one rural area of northern Vietnam. Two cohorts of pregnant women were carried out in DodaLab and FilaBavi Health and Demographic Surveillance Sites (HDSS) in Hanoi, Vietnam from April 2008 to December 2009. Together, 2515 pregnant women were identified and followed until delivery through quarterly household interviews using structured questionnaires. Almost all women delivered at health care facilities. Most of the rural women gave birth at primary health care facilities $(88.5 \%)$ while urban women primarily used secondary and tertiary hospitals (93.6\%). Caesarean section (CS) was used for 38.5\% of births in the urban area and $12.4 \%$ in the rural. Giving birth in hospitals and CS were more common among highly educated women, employed women, women living in households or communities with good economic conditions, and women expected to give birth to a son. Technology preference in delivery care was associated with better socioeconomic conditions and expecting a boy. Improving the quality and reputation of primary health care facilities, informing women about CS risks and monitoring indications of CS are important policy issues.
\end{abstract}

Keywords: Technology preference, delivery care utilization, hospital delivery, caesarean section, rural and urban, Vietnam

\section{Introduction}

Universal appropriate delivery care, focusing on delivery at health facilities and with support from skilled birth attendance, is a key strategy in order to achieve the Millennium Development Goal 5 [1]. However, the coverage of skilled birth attendance in 2008 was estimated to be $65.7 \%$ globally, with almost $100 \%$ in high income countries and only slightly above $50 \%$ in low and middle income countries (LMICs) [2].

While about 50 million women in LMICs are estimated to give birth annually in their homes without any professional help [1], birth has been concentrated in large hospitals in high income countries [3]. The choices made in relation to childbirth are influenced by the mother's wish that the delivery will be as positive and safe as possible. Nevertheless, the possible influences of other factors like economy and preference for technology have to be discussed.

Technological development is a rapidly moving process and not only patients but also physicians usually think that what is new is better. Many new technologies are useful and have improved the treatment of many diseases, but some are being overused [4]. One example is caesarean section (CS). Increased preference for and use of CS delivery, without medical indication, has been reported from several countries, high income as well as low and middle income [5,6].

Vietnam initiated the "Doi Moi" policy, which were socialistic economic reforms in 1986 and is now in a process of rapid economic development. Some segments of the society have seen their economic resources increase dramatically. Other segments have not had such benefits and the gaps are widening [7].

The national health survey in 2002 showed that $82.4 \%$ births were assisted by health workers, of those, $77.5 \%$ were deliveries at health care facilities. There were differences in the choices of place for delivery between social groups and regions [8]. Few studies of the use of delivery care have been conducted in Vietnam, and almost all of them concern rural areas [9].

The aim of the study is to investigate delivery care regarding utilization, technology preference and related factors in urban and rural areas in Vietnam.

\section{Materials and Methods}




\subsection{Study areas}

The study was conducted in two health and demographic surveillance sites (HDSS) in Hanoi, Vietnam. FilaBavi is a rural site established in 1999 with approximately 51,000 persons in 11,000 households, accounting for around $20 \%$ of the population of the district under surveillance. DodaLab, the urban site, started in 2007 covering about 38,000 persons in 11,000 households ( $12 \%$ of district's population) [10]. The aim of these HDSS is to provide basic information for health planning and policy making as well as community health training and research. In the two HDSS, a household survey is conducted every two years to collect demographic and socioeconomic information at household and individual level. Quarterly follow up surveys are carried out to update information on health and health seeking behavior and demographic information. Data are collected by 106 trained field workers (46 in FilaBavi and 60 in DodaLab) through household interview with structured questionnaires [10].

\subsection{Study design}

Two cohorts of pregnant women were conducted in parallel in the two HDSS using identical methods for data collection. In total, 2757 pregnant women were identified in the two sites during the period April 2008 to December 2009. Of those, 242 women had had abortions or had outmigrated before delivery so 2515 women were followed to delivery. Data on the use of antenatal care and delivery care were collected through quarterly household interviews using a structured questionnaire. A random sample of three percent of all pregnant women was observed or re-interviewed by field supervisors. All forms were rechecked by supervisors before being entered into the information system. Demographic and socioeconomic information about persons and households were extracted from the household surveys in the two HDSSs.

\subsection{Outcome variables}

Place of birth: at home, at commune health centers, in public hospitals at different levels or in private hospitals.

Mode of delivery: vaginal non-instrumental delivery, instrumental delivery including vacuum extraction and forceps and CS delivery with two subcategories: elective which is planned in advance before the start of labor and delivery and emergency CS.

Hospital delivery and caesarean birth were considered as technology preference in delivery care and used as dependent variables in the analyses. With this definition technology preference is influenced both by the mothers and the providers.

Birth attendant: According to WHO, "Skilled birth attendant" refers to a health professional such as a midwife, doctor or nurse, who is trained and competent in the skills needed to manage normal childbirth and the immediate postnatal period, and who can identify complications and, as necessary, provide emergency management and/or refer the case to a higher level of health care." [11].

In this study, skilled birth attendants included health providers with a formal education often on an academic level such as physicians, midwives, nurses and assistant physicians. Traditional birth attendants are excluded from skilled attendant at delivery.

\subsection{Explanatory variables}

Maternal demographic and socioeconomic characteristics include rural urban residency, mother's age, education, occupation, household economic status and community socioeconomic condition.

Household economic status was measured using wealth index composed from housing condition and asset ownership. The wealth index scores were estimated using Principal Component Analysis and classified into terciles.

Community socioeconomic condition: In DodaLab, the three communes were strategically selected as having different economic levels. In FilaBavi, three geographic types: mountainous, highland and lowland were considered as low, middle and high level, respectively regarding reported annual income and distances to the nearest health facility.

Obstetric characteristics: parity, pregnancy at high risk, use of antenatal care (ANC), sex of child. Of these, high risk pregnancy was defined as women meeting at least one of the following criteria: nullipara, aged 40 or older; multipara with four or more childbirths; women with any history of induced abortion, preterm delivery, CS, stillbirth, or neonatal death or women reporting any conditions of high blood pressure, diabetes, epilepsy or depression during pregnancy [12].

Antenatal care was defined as overall adequate when satisfying three conditions: 1) attendance to ANC during the first trimester, 2) using three or more ANC visits and 3) receiving all ANC core services at least once.

\subsection{Data analysis}

Data was entered into Access software and analyzed using STATA software version 11.0. Chi square test and ttest were used for urban-rural comparison. Factors related to the use of delivery care were analyzed separately for the urban and the rural area. Logistic regression models, both bivariate and multivariate, were used to investigate associations between outcome and explanatory variables. Multivariate models were built based on previous knowledge about factors possibly associated with delivery care and available variables in the two HDSSs.

\subsection{Ethical considerations}

The development of the two HDSS has been discussed and received support from local authorities. It was also approved by the Scientific and Ethical Committee of Hanoi Medical University and Ministry of Health of Vietnam. The participants were informed about the purpose of the study and their right to decline participation and to withdraw from the study. Verbal consent was obtained from all pregnant women. Results will only be presented on group level. Mothers can receive advice from obstetric experts within the project for the problems they have during pregnancy or regarding ANC utilization. A small gift worth 30,000 VND (less than 2 USD) was offered to each newborn baby.

\section{Results}




\subsection{Background information about the women}

The percentages of nulliparous women were $46.5 \%$ in the rural and $56.5 \%$ in the urban areas. The mean age was 26.0 in the rural area and 29.0 in the urban. Among the rural women $16.6 \%$ had graduated post high school training to be compared to $62.6 \%$ among the urban. The most common occupations were farmers in the rural and office staff in the urban area. Thirteen percent of the rural women and $10.6 \%$ of the urban were considered as high risk pregnancy. Overall adequate ANC was reported by $78.3 \%$ of the urban and $15.2 \%$ of the rural women.

Stillbirths were $0.6 \%$ of deliveries in the urban area and $0.7 \%$ in the rural. There were $17 \%$ of the urban women and $18 \%$ rural women who gave birth before 37 gestational weeks according to the reported date of the last menstrual period.

\subsection{Choices for delivery care in the two areas}

According to Table 1, nearly all women (except 5 rural women, $0.3 \%$ ) gave birth in health facilities. Most of the rural women gave birth at primary health care facilities (34.3\% at CHCs and $54.2 \%$ at district hospital) while urban women delivered mainly at secondary (36.2\%) and tertiary level facilities (57.4\%). Giving birth in hospitals was more frequent in the urban area than in the rural (97.6\% and $65.4 \%$, respectively, $\mathrm{p}<0.001$ ).

Almost all women were supported by skilled birth attendants during delivery. A larger proportion of women with non-instrumental vaginal birth were assisted by physicians in the urban area compared to in the rural area. Nurses and midwives assisted in $7.3 \%$ and $31.4 \%$ births, respectively in the urban and in the rural area. Assistant physicians attended in $26.7 \%$ of births in the rural and $0.4 \%$ births in the urban area. Traditional birth attendants assisted for 24 births, only in the rural area (1.4\%). CS and instrumental deliveries were performed by physicians. CS was performed more than three times as often for urban as for the rural women (38.5\% versus $12.4 \%$ ). About $60 \%$ of CS births in the urban and $40 \%$ of CS births in the rural areas were elective. Non-instrumental vaginal delivery was reported by $81.3 \%$ of the rural women and $57.0 \%$ of the urban women. Six point four percent of the rural women and $4.6 \%$ of the urban women were delivered by vacuum or forceps.

The mean stay in health facilities for delivery were 3.6 (95\% CI 3.5-3.8) days in the urban area and 2.7 (95\% CI 2.6-2.9) days in the rural. For delivery in provincial and central hospitals or giving birth by $\mathrm{CS}$, rural women stayed longer than urban women $(\mathrm{p}<0.001)$. A statistically significant difference in length of stay between levels of hospitals was observed only in the rural but not in the urban area.

\subsection{Factors associated with delivery care, focusing on hospital delivery and CS birth}

According to Table 2, highly educated women in both the rural and urban area were more likely to give birth in hospitals. In the rural area, giving birth in hospitals was also more frequent among women older than 35, nulliparous women, women with high risk pregnancy, women receiving overall adequate $\mathrm{ANC}$ and women who expected to give birth to a boy in the rural area.

Table 3 shows that CS was more common among women aged 35 years or more, women with high risk pregnancy or women with a better living condition in both sites. Highly educated women were more likely to have CS but a statistically significant difference could be demonstrated only in the rural areas. The same associations were found regardless of CS being used electively or as an emergency, not shown in Table 3 . The rural women with employment, women living in communities with a better socioeconomic condition or women who received overall adequate ANC had a higher probability of giving birth by CS. In the urban area, CS birth was more common among women who delivered a boy and among women living in poorer communes. The differences in CS proportion between rural and urban were similar for elective and emergency CS, not shown in Table 3 .

Table 1. Delivery Care Utilization in The Two Areas

\begin{tabular}{lcccc}
\hline & \multicolumn{2}{c}{ Rural $(\mathrm{n}=1499)$} & Urban $(\mathrm{n}=1016)$ \\
\cline { 2 - 5 } & $\mathrm{n}$ & $\%$ & $\mathrm{n}$ & $\%$ \\
\hline Place of delivery & & & & \\
CHC/Maternity house & 511 & 34.3 & 24 & 2.4 \\
District hospital & 808 & 54.2 & 20 & 2.0 \\
Provincial hospital & 145 & 9.7 & 366 & 36.2 \\
Central hospital & 19 & 1.3 & 581 & 57.4 \\
Private health facilities & 4 & 0.3 & 21 & 2.1 \\
At home & 5 & 0.3 & 0 & 0 \\
Birth attendant in vaginal non-instrumental & births & & \\
Physician & 486 & 40.1 & 535 & 92.3 \\
Midwife/nurse & 381 & 31.4 & 42 & 7.3 \\
Assistant physician & 324 & 26.7 & 2 & 0.4 \\
Traditional birth attendant & 21 & 1.4 & 0 & 0 \\
Mode of delivery & & & & \\
Vaginal non-instrumental & 1212 & 81.3 & 575 & 57.0 \\
Instrumental & 95 & 6.4 & 46 & 4.6 \\
Elective caesarean section & 74 & 5.0 & 241 & 23.9 \\
Emergency caesarean section & 110 & 7.4 & 147 & 14.6 \\
\hline
\end{tabular}

\section{Discussion}

\subsection{Preference for giving birth in hospitals at high level}

The study was conducted in the Red River Delta, the region with the highest coverage of delivery and health facilities and skilled birth attendance in Vietnam. In line with the results from the national health survey in 2002 [8], almost all women in both areas gave birth at health facilities and were assisted by health workers. This figure is much higher compared to other low and middle income countries such as India [13].

The significant difference observed between the rural and the urban areas in delivery could be expected in view of the obvious differences in availability of, and accessibility to health facilities [14]. Each commune in both areas has one commune health center (CHC). Each CHC serves for 8,500 people in FilaBavi and 13,500 people in DodaLab. There are one maternity house and several hospitals at different levels within or in close 
DOI:10.12691/ajphr-1-1-2

vicinity of DodaLab but only one district hospital in FilaBavi. However, women in FilaBavi also can access to hospitals in DodaLab within a distance of $70 \mathrm{~km}$. The average road distances to access the nearest public hospital are $1.8 \mathrm{~km}$ in DodaLab and $10.2 \mathrm{~km}$ in FilaBavi [15].

In Vietnam, the policy is that women with a low risk pregnancy should give birth at primary health care level. Basic health care services for a vaginal delivery are reported to be available at almost all communal and district health care facilities [16]. With only $4.4 \%$ women in DodaLab gave birth CHCs and district hospitals, the services at this level are not supposed to meet the perceived needs and preferences of women. There is a need for evaluation of effectiveness of CHCs in providing primary health care, including delivery care services.

Preference for care at high-level health facilities is generally recognized as the main reason for the overloading of patients at provincial and central hospitals in big cities in Vietnam. Central obstetric and gynecology hospitals had to run with $200 \%$ of their normal bed capacity. In these hospitals, deliveries accounted for $56 \%$ of the in-patients and two thirds of these were vaginal deliveries. Reasons for preference for care at high level health facilities are trust in professional qualifications of physicians, medical equipment and infrastructure as well as the quality of care at these facilities being more than that at lower levels [17].

Table 2. Factors Associated with Giving Birth in Hospitals

\begin{tabular}{|c|c|c|c|c|c|c|}
\hline & \multicolumn{3}{|c|}{ Rural } & \multicolumn{3}{|c|}{ Urban } \\
\hline & \multicolumn{6}{|c|}{ Hospital birth, n (\%) Crude OR (95\%CI) Adjusted OR (95\%CI) Hospital birth, n (\%) Crude OR (95\%CI) Adjusted OR (95\%CI) } \\
\hline \multicolumn{7}{|l|}{ Age group } \\
\hline$<25$ & $387(58.3)$ & 1 & 1 & $124(96.9)$ & 1 & 1 \\
\hline $25-34$ & $503(69.7)$ & $1.6(1.3-2.1)^{* * *}$ & $2.4(1.8-3.1)^{* *}$ & $763(97.8)$ & $1.4(0.5-4.4)$ & $1.0(0.3-3.5)$ \\
\hline $\begin{array}{l}35+ \\
\text { Education }\end{array}$ & $86(81.1)$ & $3.1(1.8-5.1)^{* * *}$ & $5.7(3.3-10.0)^{* * *}$ & $101(97.1)$ & $1.1(0.2-5.0)$ & $0.9(0.2-4.9)$ \\
\hline Secondary or less & $482(57.2)$ & 1 & 1 & $58(89.2)$ & 1 & 1 \\
\hline High school & $274(68.0)$ & $1.6(1.2-2.0)^{* * *}$ & $1.5(1.1-1.9)^{* * *}$ & $305(97.1)$ & $4.1(1.5-11.4)^{* *}$ & $3.2(1.1-9.5)^{*}$ \\
\hline \multicolumn{7}{|l|}{ Occupation } \\
\hline Employed & $247(82.9)$ & $3.1(2.2-4.3)^{* * *}$ & $1.3(0.9-1.9)$ & $646(98.5)$ & $2.6(1.2-6.0)$ & $1.2(0.4-3.6)$ \\
\hline Self employed & $729(61.1)$ & 1 & 1 & $342(96.1)$ & 1 & 1 \\
\hline \multicolumn{7}{|c|}{ Household wealth index } \\
\hline Poor & $278(57.2)$ & 1 & 1 & $298(95.2)$ & 1 & 1 \\
\hline Middle & $299(60.2)$ & $1.1(0.9-1.5)$ & $0.8(0.6-1.1)$ & $341(98.3)$ & $2.9(1.1-7.5)^{*}$ & $2.0(0.7-5.5)$ \\
\hline Rich & $390(78.5)$ & $2.7(2.1-3.6)^{* * *}$ & $1.6(1.2-2.2)^{* *}$ & $349(99.2)$ & $5.9(1.7-20.4)^{* *}$ & $3.7(1.0-14.1)$ \\
\hline \multicolumn{7}{|c|}{ Community condition } \\
\hline Low & $158(57.3)$ & 1 & 1 & $421(96.6)$ & 1 & 1 \\
\hline Middle & $568(64.6)$ & $1.4(1.1-1.8)^{*}$ & $1.3(0.9-1.7)$ & $264(98.5)$ & $2.4(0.8-7.2)$ & $2.1(0.7-6.7)$ \\
\hline High & $250(74.2)$ & $2.1(1.5-3.0)^{* * *}$ & $1.5(1.1-2.2)^{*}$ & $303(98.4)$ & $2.2(0.8-6.0)$ & $1.5(0.5-4.5)$ \\
\hline \multicolumn{7}{|l|}{ Parity } \\
\hline Nullipara & $520(74.9)$ & $2.2(1.8-2.8)^{* * * *}$ & $3.1(2.4-4.1)^{* * *}$ & $56.1(98.1)$ & $1.6(0.7-3.5)$ & $1.5(0.6-3.7)$ \\
\hline Multipara & $456(57.1)$ & 1 & 1 & $427(97.1)$ & 1 & 1 \\
\hline \multicolumn{7}{|l|}{ Pregnancy at risk } \\
\hline High risk & $146(74.9)$ & $1.7(1.2-2.4)^{* *}$ & $1.9(1.3-2.7)^{* *}$ & $105(98.1)$ & $1.3(0.3-5.6)$ & $1.5(0.3-7.0)$ \\
\hline Low risk & $830(64.0)$ & 1 & 1 & $883(97.6)$ & 1 & 1 \\
\hline \multicolumn{7}{|l|}{ ANC adequate use } \\
\hline Overall adequate & $197(82.1)$ & $2.8(2.0-3.9)^{* * *}$ & $2.0(1.4-2.9)^{* * *}$ & $721(97.8)$ & $1.4(0.6-3.2)$ & $1.0(0.4-2.4)$ \\
\hline Inadequate & $779(62.2)$ & 1 & 1 & $267(97.1)$ & 1 & 1 \\
\hline \multicolumn{7}{|l|}{ Sex of newborn } \\
\hline Boy & $559(67.5)$ & $1.2(1.0-1.5)$ & $1.3(1.0-1.6)^{*}$ & $553(97.7)$ & $1.1(1.5-2.1)$ & $1.3(0.6-3.1)$ \\
\hline Girl & $417(63.0)$ & 1 & 1 & $435(97.5)$ & 1 & 1 \\
\hline
\end{tabular}

Note: $\mathrm{p}$ values for logistic regression models: *: $\mathrm{p}<0.05 ; * *: \mathrm{p}<0.01 ; * * *: \mathrm{p}<0.001$

Table 3. Factors Associated with Caesarean Section in The Rural and The Urban Areas

\begin{tabular}{|c|c|c|c|c|c|c|}
\hline & \multicolumn{3}{|c|}{ Rural } & \multicolumn{3}{|c|}{ Urban } \\
\hline & CS birth, n (\%) & Crude OR $(95 \% \mathrm{CI})$ & $\begin{array}{c}\text { Adjusted OR } \\
(95 \% \mathrm{CI})\end{array}$ & CS birth, n (\%) & Crude OR (95\%CI) & Adjusted OR (95\%CI) \\
\hline \multicolumn{7}{|l|}{ Age group } \\
\hline$<25$ & $47(7.1)$ & 1 & 1 & $39(30.7)$ & 1 & 1 \\
\hline $25-34$ & $108(15.0)$ & $2.3(1.6-3.3) * * *$ & $2.3(1.5-2.4)^{* *}$ & $290(37.3)$ & $1.3(0.9-2.0)$ & $1.3(0.8-2.0)$ \\
\hline $35+$ & $29(27.4)$ & $5.0(2.8-8.3)^{* * *}$ & $5.6(3.1-10.1)^{* * *}$ & $59(56.7)$ & $3.0(1.7-5.1)^{* * *}$ & $2.7(1.5-4.9)^{* *}$ \\
\hline \multicolumn{7}{|l|}{ Education } \\
\hline Secondary or less & $88(10.5)$ & 1 & 1 & $19(29.2)$ & 1 & 1 \\
\hline High school & $49(12.1)$ & $1.2(0.8-1.7)$ & $1.3(0.9-1.9)$ & $126(40.3)$ & $1.6(0.9-2.9)$ & $1.7(0.9-3.3)$ \\
\hline High school + & $47(19.0)$ & $2.0(1.4-2.9)^{* * *}$ & $1.4(0.8-2.4)$ & $243(38.5)$ & $1.5(0.9-2.7)$ & $1.7(0.8-3.3)$ \\
\hline \multicolumn{7}{|l|}{ Occupation } \\
\hline Employed & $51(17.1)$ & $1.7(1.2-2.3)^{* *}$ & $1.1(0.7-1.7)$ & $256(39.1)$ & $1.1(0.8-1.4)$ & $1.1(0.8-1.5)$ \\
\hline Self employed & $133(11.2)$ & 1 & 1 & $132(37.2)$ & 1 & 1 \\
\hline \multicolumn{7}{|c|}{ Household wealth index } \\
\hline Poor & $49(10.1)$ & 1 & 1 & $104(33.3)$ & 1 & 1 \\
\hline Middle & $45(9.0)$ & $0.9(0.6-1.3)$ & $0.7(0.5-1.2)$ & $146(42.3)$ & $1.5(1.1-2.0)^{*}$ & $1.5(1.1-2.0)^{*}$ \\
\hline Rich & $87(17.5)$ & $1.9(1.3-2.7)^{* *}$ & $1.3(0.9-2.1)$ & $138(39.2)$ & $1.3(0.9-1.8)$ & $1.2(0.9-1.8)$ \\
\hline \multicolumn{7}{|c|}{ Community condition } \\
\hline Low & $26(9.4)$ & 1 & 1 & $184(42.4)$ & 1 & 1 \\
\hline Middle & $100(11.4)$ & $1.2(0.8-2.0)$ & $1.2(0.7-1.9)$ & $100(37.3)$ & $0.8(0.6-1.1)$ & $0.8(0.6-1.1)$ \\
\hline High & $58(17.2)$ & $2.0(1.2-3.3)^{* *}$ & $1.5(0.9-2.6)$ & $104(33.9)$ & $0.7(0.5-0.9)^{*}$ & $0.6(0.5-0.9)^{* *}$ \\
\hline
\end{tabular}




\section{Parity}

Nullipara

Multipara

Pregnancy at risk

High risk

Low risk

ANC adequate use

Overall adequate

Inadequate

Sex of newborn

Boy

Girl

$85(12.2)$

99 (12.4)

37 (19.2)

147 (11.3)

51 (21.2)

133 (10.6)

$$
1.0(0.7-1.3)
$$

1

$1.9(1.3-2.8)^{* * *}$

1

$2.8(2.0-3.9) * * *$

1

$101(12.2)$

$83(12.5)$

$1.0(0.7-1.3)$

\section{$1.3(0.9-2.1)$ \\ $205(36.0)$}

1

1

$1.67(1.1-2.5)^{*}$
183 (41.6)

53 (49.5)

335 (37.1)
$282(38.3)$

$106(38.8)$
$0.8(0.6-1.0)$

1

$1.7(1.1-2.5)^{*}$

$1.0(0.7-1.3)$

1

245 (43.5)

$143(32.1)$
$1.6(1.3-2.1)^{* * * *}$
A similar concentration of births in higher level hospitals has been found in other countries such as Finland [3]. In FilaBavi, the percentage of hospital deliveries was also reported to increase from around $30 \%$ in 1999 to more than $60 \%$ in 2009, especially among women with better economic status. This tendency will further widen the inequality in the use of delivery care and exacerbate the overload in hospitals. Improving the quality and reputation of primary delivery care facilities is needed.

Of course, women can follow their preference only if the option is available. The urban women gave birth at provincial and central hospitals might simply be the utilization of available recourses. However, the choice to give birth in high-level hospitals can signify prestige, reflecting an ambition to show the availability of economic resources sometimes, and is possibly often accompanied by the perception that high quality care is the same as high-level care, both institutionally and professionally. A qualitative study in Scotland showed that the choice of place of birth is a complex decision that women made based primarily on their perceptions of safety and inter-related socio-economic factors. The choice made by women is also influenced by the presentation of choice by health professionals, social networks, pregnancy complications and geographic accessibility [18].

In the urban setting, women prefer to give birth in secondary and tertiary hospitals. This may be because the facilities are in close vicinity and are supposed to have a better quality of services. When geographic accessibility is not a factor, perceptions of safety might be important for their choice. According to Government policy, women are allowed to have only one or two children so they try to have the best possible care for their newborn. The women with better economic conditions can afford and are willing to pay for maternity services available at central and provincial hospitals in urban areas.

However, prestige can also be perceived as a factor influencing delivery choices. The ambition to show that economic resources are available sometimes, possibly often, goes together with the perception that high quality care means care with high quality, institutionally and professionally. For medical indications, giving birth in hospitals at higher levels is generally supposed to imply longer stay. As the duration for delivery was similar in different levels of hospital in the urban area in this study, this may be evidence for preference according to prestige.

The urban women, who gave birth in hospitals, also received assistance from physicians more often. The increased use of skilled birth attendant is mainly due to the increased involvement of physicians as seen also in other developing countries [1]. However, physician birth attendants were also common in the rural area, where there is a lack of physicians. The physician attendance preference might also exacerbate the shortage of doctors at primary health care level. There is a need to strengthen the role of midwives in delivery care provision through education and information to mothers.

\subsection{CS birth preference}

Giving birth more frequently in hospitals, urban women also had more CS delivery than the rural women. The finding is in line with a recent WHO survey, in which Vietnam had $35.6 \%$ CS deliveries. Among nine Asian countries, Vietnam had the second highest rate of CS birth, only lower than China with $46.2 \%$ [6]. In many countries, $\mathrm{CS}$ is reported to be increasing with the improvement of socioeconomic conditions and development of medical technology. A large proportion of the increase is supposed to be due to the preference of the women and to be without medical basis [19].

The percentage of CS birth in the urban area was much higher than the expected rate of medically indicated CS, i.e. about 10-15\% [19] suggesting overuse. In Vietnam, CS is not availbale at CHCs, but in hospitals. CS is available in many hospitals that can easily access in the urban area. The self-reported nature of data in this study decreases the possibility to assess if CS was medically indicated or not. However, a percentage as high as almost $40 \%$ of urban women using CS cannot possibly be solely due to medical reasons.

$\mathrm{CS}$ with medical indication is supposed to need a longer duration of hospital stay and is therefore more expensive than CS without medical indication and vaginal delivery. The smaller differences between average cost and length of hospital stay for CS and vaginal delivery in the urban area than those in the rural suggests to some extent that a substantial proportion of the CS in the urban area have been performed without medical justification. A community-based study in Hanoi (2008) showed that $30.3 \%$ of all deliveries were performed by a CS and $14 \%$ of all CS births was not medically indicated [20].

According to Bowling, "preference is the expression of a value for alternative options for action after informed deliberation of risks and benefits". Decision for a treatment should be made considering and understanding risks, patient and professional preferences [21]. Of course, the benefit of CS use when needed as in obstructed labor is understandable. In low income countries, lack of availability of, or access to, maternal health services and the corresponding underuse of CS are factors predisposing to high maternal and perinatal mortality [5]. CS therefore should be more and more available in rural areas to prevent maternal and neonatal deaths in these countries [22].

However, higher CS rates than the optimum in many high income countries do not confer additional health gain 
but may increase maternal risks [5]. Elective CS without medical indication has been shown to increase the risk of maternal and neonatal illness and death, infection, pain, poor birth experience, delayed contact with the baby, increased length of hospital stay and readmission [6,23].

When deciding to have elective CS, the women might not know or may not consider the risk for immediate and long-term surgically unwanted consequences for them and their babies. A possible reason is that sufficient information may not be supplied by providers as a part of ANC [23]. In one previous study, we found that only $23.6 \%$ of the rural women received antenatal health consultation [10]. Increasing women's knowledge about advantages and disadvantages of CS through better communication during antenatal care might contribute to a reduction in the number of CS without medical indications.

There can be many other reasons for the request of a mother for CS such as: fear of pain, assumed safety for child, expected faster recovery after delivery and better sexual life. The women can also have a wish to deliver on a "good day" according to cultural beliefs [23]. In Asian countries, the partner's preference as well as that of the mother in law, also significantly influenced their choice [24].

Maternal request for $\mathrm{CS}$ can be patient driven but women's preferences might be also influenced by physicians' attitudes [25]. Physicians often suggest CS to their patients as the safe mode of delivery from their perspective [26]. This is very important in the Vietnamese context where the physician-patient relation is a hierarchical relationship where women normally follow suggestions by the physicians.

Physicians tend to agree to a CS by the request of women [27]. The reasons can be fear of litigation as in some other countries and because it saves time, more convenient and also gives more money to the hospital [23,27]. In Vietnam, CS not only saves time and reduces the time for monitoring of vaginal delivery under pressure of work overload but. It is necessary to strengthen compliance with medical indications of CS and at the same time, guidelines for practice of caesarean delivery on maternal request are needed. In the long-term, solutions to solve overloading at provincial and central hospitals can contribute to a reduction in $\mathrm{CS}$ without medical indications.

\subsection{Factors associated with user technology preference}

In this study, CS birth and giving birth in hospitals, especially at provincial and central levels, are related to each other and associated with better socioeconomic conditions. As found also in other studies [14], hospital delivery was more common among highly educated women, employed women or women with a better living standard, especially in the rural area. These women were also more likely to give birth using CS. Associations between high education and good economy and CS preference without medical indications have been reported earlier [28]. Good socioeconomic conditions of communities also increased the risk of CS due to better accessibility to health facilities [29].

Nulliparous women, older women or women with high risk pregnancy have been reported to give birth more frequently in hospitals [3]. In this study, observation in the rural but not in the urban area indicated that most women used and to some extent overused care at hospital level. The percentage of CS birth also increased with age [30]. Women who received adequate use of ANC more frequently gave birth in hospitals and were more often assisted by physicians. They usually had better socioeconomic conditions and closer contact with health workers through ANC utilization [14].

The importance of having a son might be reflected in delivery practice. Hospital delivery was more common among rural women who delivered a boy. To our knowledge similar findings have not been reported previously. The finding can be an expression of the preference for sons in Vietnam. No corresponding significant difference could be seen in the urban area since the percentage of hospital delivery is generally close to $100 \%$.

In the urban area, women who delivered a boy also had a higher rate of CS birth. The more frequent CS in the delivery of boys suggests "son preference" behavior and sex selection, reported previously in Vietnam [31]. More than $80 \%$ of pregnant women in the present study reported that they knew the sex of the fetus before delivery. This has been found to be related to the extensive use of prenatal ultrasound [32], despite the fact that doctors are not allowed to inform pregnant women about the sex of the fetus. The women might believe that CS is better for the child and therefore should be preferred for boys. To discuss the phenomenon more in-depth and the effects of son preference is not an ambition of this study.

The study was conducted in two specific areas where the socioeconomic condition is comparatively good and the health care system is supposed to be better compared to other regions of the country. The results therefore cannot be generalized. However, it may be of relevance for local policy, strategy and intervention to other areas with similarity regarding demography, economy, health care structure et cetera. The women's preference of giving birth in hospitals and CS in the urban areas might occur later in rural areas. Further studies on influences from other household members and from providers on women's choices are also needed for policy making.

\section{Conclusion}

The coverage of delivery care was high and women's choices were made based on the available health care resources and technology preference. The women in urban areas gave birth more often at high level health care facilities with more assistance from physicians and more CS. In urban area there was an overuse of hospital care for giving birth and the use of CS, which might partly be due to perceived prestige by the mothers. Technology preference in delivery care was associated with better socioeconomic conditions and expecting a boy. Improving the quality and reputation of primary health care facilities, improving the role of midwives in delivery care provision, informing women about CS risks and monitoring indications of CS during pregnancy are important issues to pursue in future policies.

\section{Acknowledgement}


This research is supported by grants from Sida/SAREC and the Nordic School of Public Health, Sweden. We would like to thank Professor Max Petzold, Professor Nguyen Duc Hinh for his useful contributions to supervision of the study, interpretation of the data and revising the manuscript. We are grateful to all field workers and pregnant women at FilaBavi and DodaLab for their contribution to data collection and participation.

\section{References}

[1] Stanton, C., et al., Skilled care at birth in the developing world: progress to date and strategies for expanding coverage. Journal of biosocial science, 2007. 39(1): p. 109-20.

[2] Barona-Vilar, C., et al., Perceptions and experiences of parenthood and maternal health care among Latin American women living in Spain: A qualitative study. Midwifery, 2012.

[3] Hemminki, E., A. Heino, and M. Gissler, Should births be centralised in higher level hospitals? Experiences from regionalised health care in Finland. BJOG : an international journal of obstetrics and gynaecology, 2011. 118(10): p. 1186-95.

[4] Moser, M., Comments on progress, medical care, and the overuse of technology. Journal of clinical hypertension, 2005. 7(8): p. 4424.

[5] Betran, A.P., et al., Rates of caesarean section: analysis of global, regional and national estimates. Paediatr Perinat Epidemiol, 2007. 21(2): p. 98-113.

[6] Lumbiganon, P., et al., Method of delivery and pregnancy outcomes in Asia: the WHO global survey on maternal and perinatal health 2007-08. Lancet, 2010. 375(9713): p. 490-9.

[7] Vietnam Ministry of Health, Joint Annual Health Review 2010, 2011, Ministry of Health $(\mathrm{MoH})$,: Hanoi.

[8] Vietnam Ministry of Health and Vietnam General Statistics Office, Results of the National Health Survey 2001-2002.2003, Hanoi: Medical Publishing House.

[9] Sepehri, A., et al., How important are individual, household and commune characteristics in explaining utilization of maternal health services in Vietnam? Social science \& medicine, 2008. 67(6): p. 1009-17.

[10] Tran, T.K., et al., Urban - rural disparities in antenatal care utilization: a study of two cohorts of pregnant women in Vietnam. BMC health services research, 2011. 11: p. 120.

[11] WHO, Fifty-Seventh World Health Assembly. Provisional agenda item 12.10 A57/13, 2004, WHO: Geneva.

[12] Dangal, G., High-Risk Pregnancy. The Internet Journal of Gynecology and Obstetrics, 2007. 7:1.

[13] Pathak, P.K., A. Singh, and S.V. Subramanian, Economic inequalities in maternal health care: prenatal care and skilled birth attendance in India, 1992-2006. PloS one, 2010. 5(10): p. e13593.

[14] Gabrysch, S. and O.M. Campbell, Still too far to walk: literature review of the determinants of delivery service use. BMC pregnancy and childbirth, 2009. 9: p. 34.

[15] Tran, T.K., et al., Factors associated with antenatal care adequacy in rural and urban contexts-results from two health and demographic surveillance sites in Vietnam. BMC health services research, 2012. 12: p. 40

[16] Vietnam Ministry of Health, National plan on safe motherhood in Vietnam 2003-2010, M.o.H. (MoH), Editor 2003, Medical Publishing House, Hanoi. p. 45-56.

[17] Le, Q.C., et al., Hospital overload in Hanoi and Ho Chi Minh City - An assessment and recommendations, 2007, Health Strategy and Policy Institute: Hanoi.

[18] Pitchforth, E., et al., "Choice" and place of delivery: a qualitative study of women in remote and rural Scotland. Quality \& safety in health care, 2009. 18(1): p. 42-8.

[19] Mazzoni, A., et al., Women's preference for caesarean section: a systematic review and meta-analysis of observational studies. BJOG : an international journal of obstetrics and gynaecology, 2011. 118(4): p. 391-9.

[20] Nguyen, T.T.T. and H.D. Nguyen, Caesarean section and related factors in Gia Lam and Hoan Kiem districs, Hanoi 2007. Medical and Pharmatical Information Journal, 2008. 2008(11): p. 23-25.

[21] Bowling, A. and S. Ebrahim, Measuring patients' preferences for treatment and perceptions of risk. Quality in health care : QHC, 2001. 10 Suppl 1: p. i2-8.
[22] Alkire, B.C., et al., Obstructed labor and caesarean delivery: the cost and benefit of surgical intervention. PloS one, 2012. 7(4): p. e34595.

[23] Collard, T.D., et al., Elective cesarean section: why women choose it and what nurses need to know. Nursing for women's health, 2008. 12(6): p. 480-8.

[24] Pang, M.W., et al., A longitudinal observational study of preference for elective caesarean section among nulliparous Hong Kong Chinese women. BJOG : an international journal of obstetrics and gynaecology, 2007. 114(5): p. 623-9.

[25] Jacquemyn, Y., F. Ahankour, and G. Martens, Flemish obstetricians' personal preference regarding mode of delivery and attitude towards caesarean section on demand. European journal of obstetrics, gynecology, and reproductive biology, 2003. 111(2): p. 164-6.

[26] Hantoushzadeh, S., et al., Caesarean or normal vaginal delivery: overview of physicians' self-preference and suggestion to patients. Archives of gynecology and obstetrics, 2009. 280(1): p. 33-7.

[27] Habiba, M., et al., Caesarean section on request: a comparison of obstetricians' attitudes in eight European countries. BJOG : an international journal of obstetrics and gynaecology, 2006. 113(6): p. 647-56.

[28] Ronsmans, C., S. Holtz, and C. Stanton, Socioeconomic differentials in caesarean rates in developing countries: a retrospective analysis. Lancet, 2006. 368(9546): p. 1516-23.

[29] Leone, T., S.S. Padmadas, and Z. Matthews, Community factors affecting rising caesarean section rates in developing countries: an analysis of six countries. Social science \& medicine, 2008. 67(8): p. 1236-46.

[30] Chu, K.H., et al., Women's preference for cesarean delivery and differences between Taiwanese women undergoing different modes of delivery. BMC health services research, 2010. 10: p. 138

[31] Belanger, D., Son preference in a rural village in North Vietnam. Studies in family planning, 2002. 33(4): p. 321-34.

[32] Sufang, G., et al., Delivery settings and caesarean section rates in China. Bulletin of the World Health Organization, 2007. 85(10): p. 755-62. 